\title{
Bone substitutes in Le Fort I osteotomy to promote bone union and skeletal stability
}

\author{
L.Zanettini, W.Polido, R.Pagnoncelli
}

Background: Maxillary advancement by Le Fort I osteotomy has become the standard procedure to restore function and facial aesthetics, correct skeletal and occlusal discrepancies and treat obstructive sleep apnea in patients with facial deformities. Incomplete ossification between the bone segments at the jaw osteotomy site has proven to be a major problem in these cases. There are several studies in the literature that address orthognathic surgery, but only a limited number that discuss the use of graft materials in maxillary osteotomy. Bone grafts were introduced in recent decades in order to promote and improve bone union and prevent the formation of gaps.

Objectives: This study will discuss the results obtained using bone substitutes in orthognathic surgery for maxillary advancement and maxillary repositioning with Le Fort I osteotomy, in a case report and a literature review, covering the main indications and specifying the type of material used and the stability of the surgical procedure postoperatively.

Methods: A 21-year-old patient, EMRS, was seen at the Oral and maxillofacial surgery and traumatology service at the Pontifícia Universidade Católica do Rio Grande do Sul Department of Dentistry (Brazil) with functional and esthetic complaints. Intra-oral clinical examination, whole face tomography and facial analysis found the patient to have a facial deformity classified as Angle Class III, with transverse

This is the author's manuscript of the work published in final form as:

Zanettini, L., Polido, W., \& Pagnoncelli, R. (2019). Bone substitutes in Le Fort I osteotomy to promote bone union and skeletal stability. International Journal of Oral and Maxillofacial Surgery, 48, 262-263. https://doi.org/10.1016/j.ijom.2019.03.802 
occlusal plane abnormality, deviation of the facial midline, maxillary atresia and malocclusion, causing loss of masticatory function and facial disharmony. (The treatment option recommended to correct this deformity was bilateral maxillary orthognathic surgery. Surgical planning included surgery on models and predictive cephalometric plots, plus facial analyses and virtual plotting using digital software to simulate the final post-surgical result. The surgical plan defined a $5 \mathrm{~mm}$ maxillary advancement, $1 \mathrm{~mm}$ maxillary intrusion on the left side and $2 \mathrm{~mm}$ maxillary extrusion on the right side, accomplished by Le Fort I maxillary osteotomy. The plan also called for rotation of the mandible, after the bilateral sagittal osteotomy, and refitting into the operated maxilla. It was predicted that after rigid fixation of the maxilla there would be a gap between the osteotomy bone segments, and so a bone graft with biomaterial, bovine bone substitute embedded in collagen (Bio- Oss Collagen - BOC), was planned to promote bone union and to improve three-dimensional skeletal stability. The bone graft was planned for the maxilla on the patient's right side, since, according to the surgical plan, the bone gap- would be larger on this side.

Findings: The surgical procedure was conducted with general anesthesia, according to the recommended procedure. On the patient's right side, where the osteotomy site gap was larger, in addition to the fixing plates and screws, the BOC was grafted $(500 \mathrm{mg})$. The left side was not grafted, just fixed with plates and screws. The graft material was moistened with sterile saline solution, allowing it to be shaped to the correct required shape and then applied in direct contact with the bone walls along the osteotomy line. (Fig. 3A, 3B) The incision was sutured by planes, first the internal plane and then the mucosa, both with absorbable VicrylTM 4-0 thread (poliglactina 910). Two months after surgery, the patient underwent full face tomography (axial, coronal, and sagittal slices and 3D reconstruction). The maxillary and mandibular bone segments can be seen fixed in position with plates and screws and on the right side of the maxilla, where the BOC graft was placed, there is localized thickening of the bone, compatible with bone neoformation. 
Conclusion: In conclusion, based on the clinical and tomographic results of this study, the bone substitute used proved to be an adequate graft material for use in Le Fort I osteotomies to promote bone union. While these results are encouraging, more studies are needed to evaluate whether the material is as effective as autogenic bone for preventing relapses and promoting bone union in maxillary advancement and repositioning, and also in other orthognathic surgery procedures. 